\title{
An easy and practical prognostic parameter: tumor-stroma ratio in Luminal, Her2, and triple-negative breast cancers
}

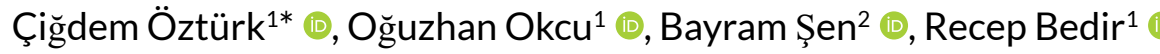

\begin{abstract}
SUMMARY
OBJECTIVE: The stroma surrounding the tumor cells is important in tumor progression and treatment resistance, besides the properties of tumor cells. Studies on the tumor stroma characteristics will contribute to the knowledge for new treatment approaches.

METHODS: A total of 363 breast cancer patients were evaluated for the tumor-stroma ratio. The percentage of stroma was visually assessed on hematoxylin-eosin stained slides. The cases of tumor-stroma ratio more than $50 \%$ were categorized as tumor-stroma ratio high, and those less than 50\% and below were categorized as tumor-stroma ratio low.

RESULTS: Tumor-stroma ratio-high tumors had shorter overall survival ( $p=0.002$ ). Disease-free survival tended to be shorter in tumor-stroma ratiohigh tumors ( $p=0.082$ ) compared with tumor-stroma ratio-low tumors. Tumor-stroma ratio was an independent prognostic parameter for the total group of patients $(p=0.003$ ) and also axillary lymph node metastasis and tumor-stroma ratio was statistically associated $(p=0.004)$. Also, tumorstroma ratio was an independent prognostic parameter in node-positive Luminal A and B subgroups for overall survival ( $p<0.001)$.

CONCLUSION: Tumor-stroma ratio is an independent prognostic parameter that can be evaluated quite easily in all molecular subtypes of all breast cancers and does not require extra cost and time to evaluate.

KEYWORDS: Stromal Tumor. Breast neoplasms. Survival. Prognosis.
\end{abstract}

\section{INTRODUCTION}

Globally, determining the biological behavior of breast carcinomas (BC), which are the most common cause of cancer deaths in women, is expected to produce essential knowledge for developing new therapeutic approaches ${ }^{1}$. Despite the ever-increasing knowledge accumulation, 30\% of patients still develop recurrence after treatment. Therefore, it is thought that not only tumor cells, whose properties are well-known in many respects, but also the stroma surrounding the tumor cells are important in the progression and treatment resistance of the tumor $^{2,3}$. It is a complex issue that how tumor stroma and its components follow in determining tumor behavior. It is thought that tumor stroma affects tumor progression by being affected by a cell to cell, cell to extracellular matrix, genetic, physiological, and environmental factors ${ }^{4}$. Studies on the characteristics of stroma surrounding the tumor cells will contribute to the knowledge for new treatment approaches ${ }^{5}$.

Many different components contribute to tumor stroma. It is not always practical to evaluate these components separately, but even determining the tumor-stroma ratio (TSR) gives information about the prognosis of patients. It has been reported that the amount of tumor stroma is an independent prognostic parameter in many tumors ${ }^{6-9}$. In our study, the relation of TSR with prognostic parameters and survival was evaluated in both triple-negative (TN) and ER-positive BC. While the chemotherapy option of patients with TN and PT1/PT2 N0 is controversial, lymph node-positive patients are candidates for adjuvant chemotherapy ${ }^{10}$. We also investigated the role of TSR in survival in these node-positive Luminal A and B groups.

\section{METHODS}

\section{Histopathological scoring}

The TSR was visually evaluated as previously described by Mesker et al. ${ }^{11}$. The original $4 \mu \mathrm{m}$ routine hematoxylin and eosin (H\&E)-stained slides from formalin-fixed paraffin-embedded blocks of the primary tumor were assessed by conventional light microscopy (Olympus, BX-51, ocular $22 \mathrm{~mm}$ ). The area with the highest tumor stroma was determined in the $4 \times$ objective. The most stroma-abundant area on the slide in which tumor

\footnotetext{
${ }^{1}$ Recep Tayyip Erdoğan University Training and Research Hospital, Pathology Department - Rize, Turkey.

${ }^{2}$ Recep Tayyip Erdoğan University Training and Research Hospital, Biochemistry Department - Rize, Turkey.

*Corresponding author:dr.ozturkcigdem@gmail.com

Conflicts of interest: the authors declare there is no conflicts of interest. Funding: none.

Received on November 30, 2021. Accepted on December 05, 2021.
} 
cells can be seen on each side was scored using a 10× objective (north-east-southwest). The TSR was scored in multiples of 10 per image field (e.g., 10, 20, and 30\%). The cases of TSR more than $50 \%$ were categorized as TSR high, and those less than $50 \%$ and below were categorized TSR low. Necrosis, in situ tumors, mucus-secreting tumor areas, previous biopsy areas, and peripheral sides of tumors were excluded in evaluating the TSR. Representative examples of microscopic fields selected for TSR quantification from TSR-high and TSR-low tumors are shown in Figures 1 and 2.

Evaluation of the TSR was assessed successfully in all the tumors (100\%). Cohen's kappa coefficient revealed an almost perfect agreement in classification (kappa $=0.85$; $94 \%$ concordance in classification) for a set of tumors scored by both observers (ÇÖ and OO).

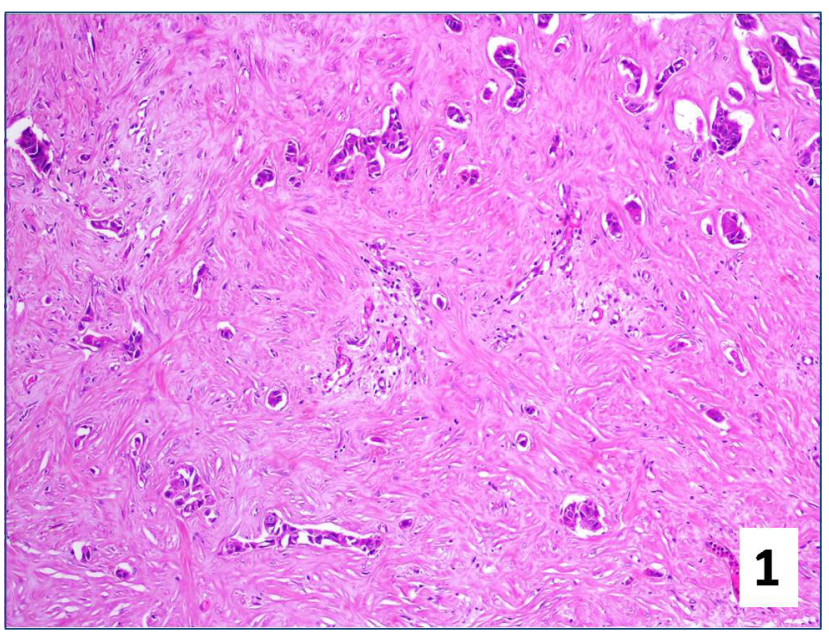

Figure 1. Example of stroma-rich (stroma ratio $\geq 50 \%$ ). Hematoxylin and eosin-stained $4 \mu \mathrm{m}$ sections of primary breast tumors (original magnification $\times 100$ ).

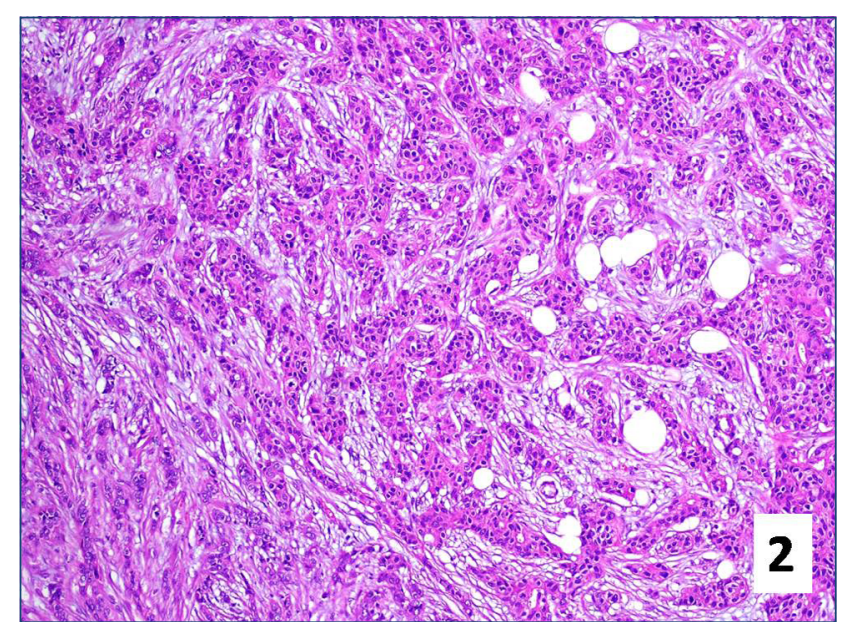

Figure 2. Example of stroma-poor (stroma ratio <50\%). Hematoxylin and eosin-stained $4 \mu \mathrm{m}$ sections of primary breast tumors (original magnification $\times 100$ ).

\section{Clinical and demographic data and tissues}

We selected the patients with invasive breast cancer between 2010 and 2020 from the database of our hospital. Patients who received neoadjuvant chemoradiotherapy, who had distant organ metastasis at the time of diagnosis, who died due to post-op complications in the first month after surgery, whose clinical data could not be reached, who were not followed up after the operation in our hospital, whose hormone profile was not interpreted, who were out of follow-up for any reason, and whose HE slides and formalin-fixed paraffin-embedded blocks could not be found in our archive were excluded from the study.

The clinical information was obtained from retrieving the medical records, including gender, age, histological tumor type, grade, tumor size, lymph node status, type of surgery, and patient follow-up information. All cases were divided into molecular subtypes based on the ER, PR, HER2, and Ki-67 immunohistochemical staining patterns and histological types, according to the classification of breast cancer by World Health Organization ${ }^{2,12}$.

As a result, 363 patients were included in the study, and the relationship between TSR, and clinicopathological parameters, disease-free (DFS), and overall survival (OS) were investigated.

\section{Statistical analysis}

Statistical analysis was performed using SPSS version 21 software. Kolmogorov-Smirnov test, histogram graphics, Mann-Whitney U test, chi-square, Fisher's exact test, Hosmer-Lemeshow test, log-rank test, Cox regression, and Kaplan-Meier survival analysis were performed. For statistical significance, the p-value was accepted as $<0.05$.

\section{RESULTS}

\section{Tumor-stroma ratio and clinical and pathological parameters}

Tumor-stroma ratio could be evaluated in a total of 363 patients, 2 of whom were men. The median age of patients was 55 (25-100) years, and the median follow-up time was 46 (2-132) months. A total of 324 of the cases had invasive ductal carcinoma (IDC), of whom 20 had invasive lobular carcinoma (ILC) and 19 patients had other histological types. When the cases were evaluated according to the $T$ stages, 146 cases were found in T1, 196 cases were in T2, and 21 cases were found in the T3 stage. According to the molecular subtypes, 135 cases were Luminal A, 160 cases Luminal B, 30 cases Her2, and 32 cases were in the triple-negative group.

There was no statistical relationship between TSR and clinicopathological parameters such as age, hormone status, molecular 
and histological type, and angiolymphatic invasion. Among the clinicopathological parameters, there was a statistically significant difference between axillary lymph node metastasis and TSR $(\mathrm{p}=0.004)$. Accordingly, as the TSR increased, the incidence of axillary lymph node metastasis increased. The main characteristics of the included studies are listed in Table 1.

Table 1. Correlations between tumor-stroma ratio and well-established prognostic factors.

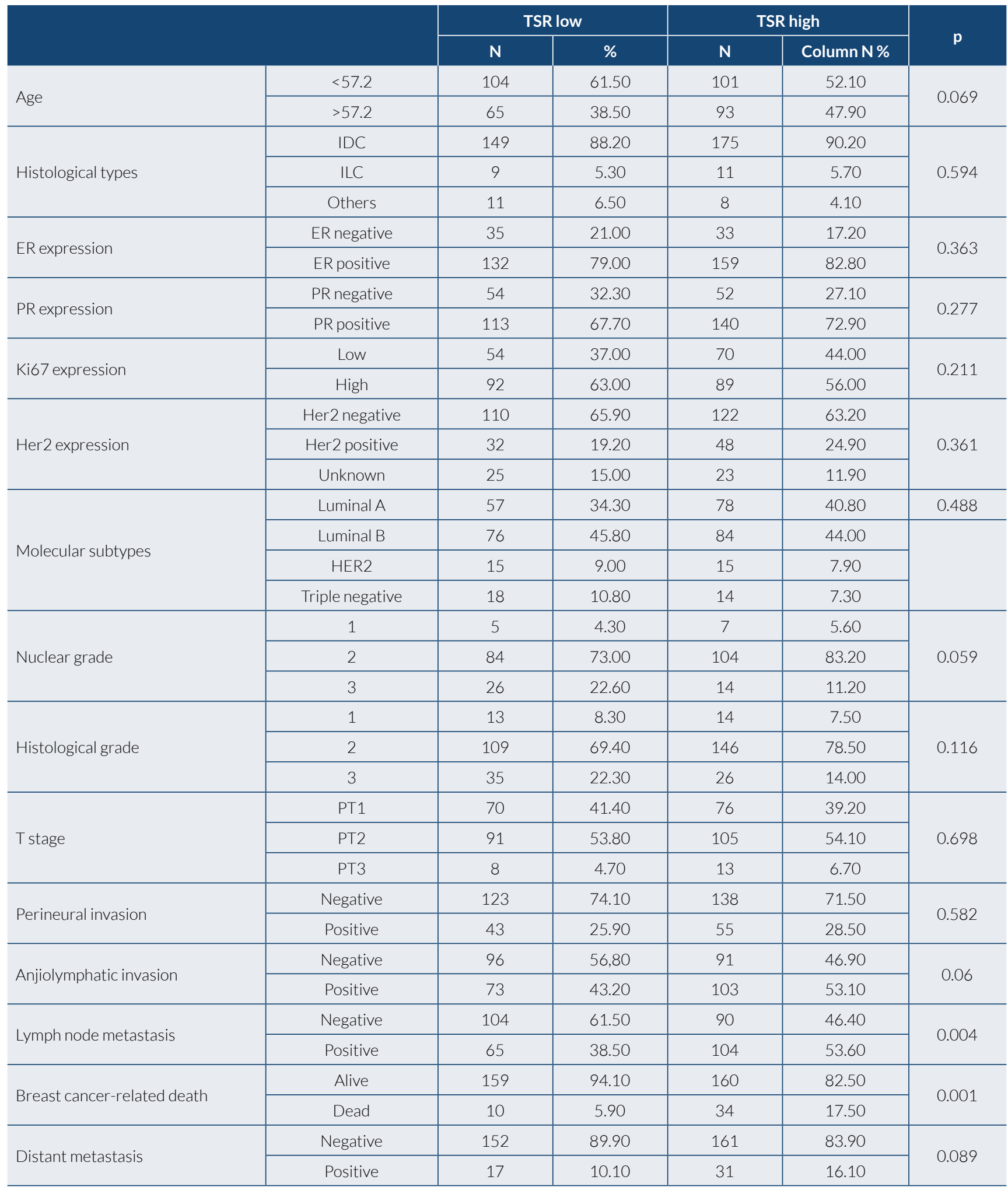




\section{Tumor-stroma ratio and prognostic associations with outcome}

A total of 194 (54\%) patients were classified as TSR high and $169(46 \%)$ patients as TSR low. OS was found for patients with TSR-high tumors as compared to patients with TSR-low tumors in univariate analysis $(\mathrm{p}=0.003)$. In multivariate analysis, the TSR was an independent prognostic variable for OS (Table 2).

In univariate analyses $(\mathrm{p}=0.86)$, the TSR was not an independent prognostic variable for DFS. After correction for the factors also used in multivariate analyses for DFS, no significant difference was obtained.

Molecular subtypes and axillar lymph node metastasis were independent prognostic variables for DFS in multivariate analysis. Even performing Kaplan-Meier curve for OS showed a significant difference between TSR-high and TSRlow patients ( $\mathrm{p}=0.002$ ) (see Figure 3 ). A trend was seen toward a worse DFS for patients with TSR-high tumors compared to patients with TSR-low tumors in the Kaplan-Meier curve $(\mathrm{p}=0.082)$ (see Figure 4).

A total of 104 (61.5\%) node-positive Luminal A and B breast carcinoma patients were classified as TSR high and 65

Table 2. Cox univariate and multivariate analysis for overall survival of all patients.

\begin{tabular}{|c|c|c|c|}
\hline \multirow{2}{*}{ Variables } & Univariate & Multivariate & \multirow{2}{*}{$\begin{array}{c}\mathrm{HR} \\
(95 \% \mathrm{Cl})\end{array}$} \\
\hline & $p$ & $p$ & \\
\hline Tumor-stroma ratio & 0.003 & 0.042 & $\begin{array}{c}2.381 \\
(1.033- \\
5.485)\end{array}$ \\
\hline Metastasis & $<0.001$ & $<0.001$ & $\begin{array}{c}7.038 \\
(2.917- \\
16.978)\end{array}$ \\
\hline ER expression & 0.012 & & \\
\hline PR expression & 0.02 & & \\
\hline Her2 expression & 0.031 & & \\
\hline Ki67 proliferation index & 0.015 & & \\
\hline Molecular subtypes & $<0.001$ & 0.001 & $\begin{array}{c}10.382 \\
(2.731- \\
39.467)\end{array}$ \\
\hline Nuclear grade & 0.223 & & \\
\hline Histological grade & 0.079 & & \\
\hline Tumor size & 0.013 & & \\
\hline Age & $<0.001$ & 0.008 & $\begin{array}{c}1.035 \\
(1.009- \\
1.062)\end{array}$ \\
\hline Lymph node metastasis & $<0.001$ & 0.025 & $\begin{array}{c}3.945 \\
(1.187- \\
13.110)\end{array}$ \\
\hline Angiolymphatic invasion & $<0.001$ & & \\
\hline Perineural invasion & 0.375 & & \\
\hline
\end{tabular}

(38.5\%) patients as TSR low. OS was found for these patients with TSR-high tumors as compared to patients with TSR-low tumors in univariate analysis (p:0.003). In multivariate analysis, the TSR was an independent prognostic variable for OS [Hazard ratio (HR) 5.33; 95\%CI 1.224-23.203; $\mathrm{p}=0.026$ ]. Patients with TSR-high node-positive Luminal A and B tumors show a significantly worse overall survival compared to patients with TSR-low tumors in the Kaplan-Meier curve $(\mathrm{p}<0.001)$ (see Figure 5). A trend was seen toward a worse DFS for patients with TSR-high tumors compared to patients with TSR-low tumors in the Kaplan-Meier curve ( $\mathrm{p}=0.066$ ) (see Figure 6).

\section{DISCUSSION}

The relation of TSR with survival status was first investigated by Mesker et al. They found that patients with TSR more than $50 \%$ showed significantly worse OS and DFS. They suggested that TSR could serve as an independent parameter for predicting clinical outcomes in early-stage colon cancer ${ }^{11}$.

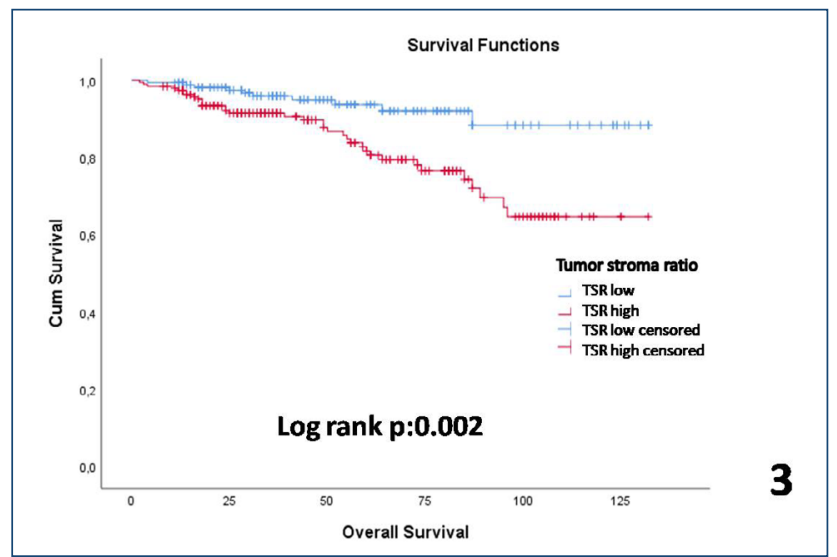

Figure 3. Kaplan-Meier curves for tumor-stroma ratio for the total patient population. Patients with TSR-high tumors show a significant overall survival compared to patients with TSR-low tumors.

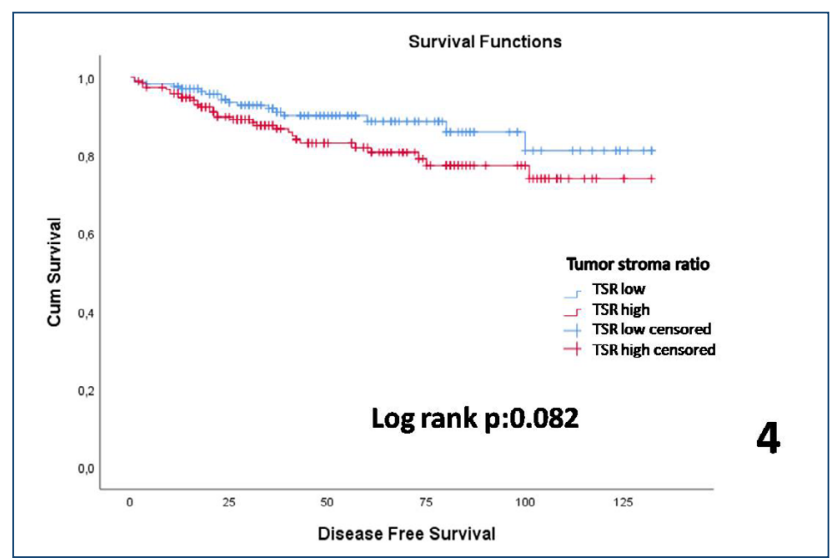

Figure 4. A trend was seen toward a worse disease free survival for patients with TSR-high tumors compared to patients with TSR-low tumors. 
The tumor stroma consists of fibroblasts, pericytes, bone marrow-associated mesenchymal stem cells, adipocytes, macrophages, and immune cells ${ }^{13}$. These components play a role in neoangiogenesis, metastasis, and tumor progression ${ }^{14}$. It is not always morphologically possible to evaluate these components separately. Additional studies may be needed to evaluate these components; however, TSR can only be evaluated by light microscopy.

Routine pathology reports include the following parameters that affect patient prognosis, such as nuclear and histological grade, molecular subtype, lymph node metastasis, and lymphovascular invasion ${ }^{15}$. In addition to these parameters, the TSR, an independent prognostic indicator with many studies, is a convenient and valuable parameter for patient prognosis.

Studies in the literature evaluate stroma in BC using digital methods such as machine learning algorithms and automated analyses ${ }^{9}$. The advantage of digital methods over light microscopy is to evaluate whole tumor tissue with digital methods, while a more limited area is evaluated in light microscopy. While

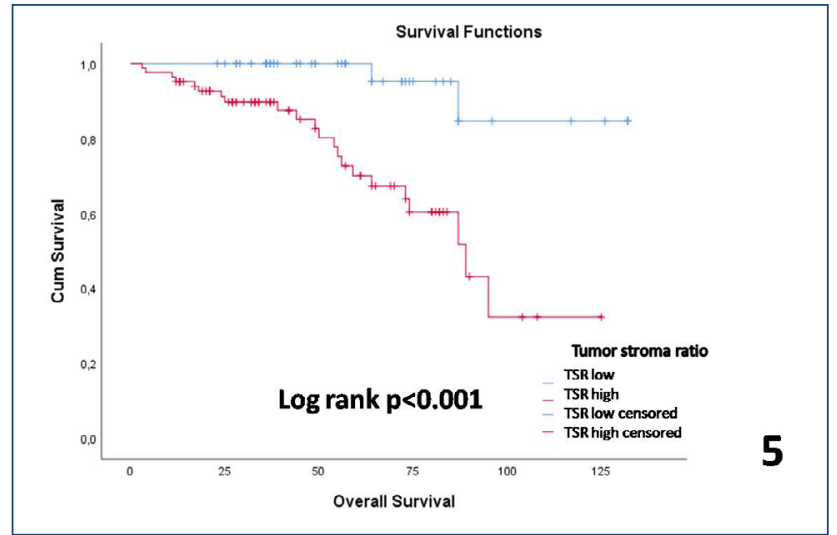

Figure 5. Kaplan-Meier curves for tumor-stroma ratio for nodepositive Luminal A and B tumors. Patients with TSR-high tumors show a significant overall survival compared to patients with TSR-low tumors.

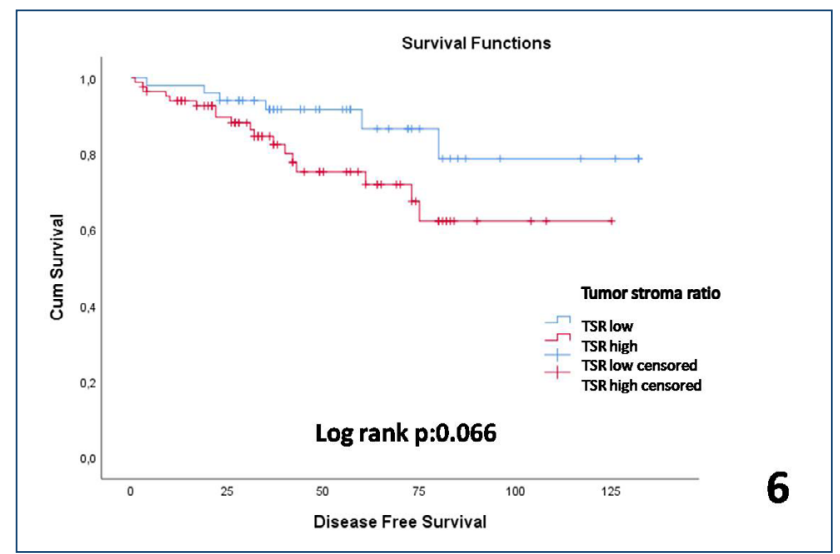

Figure 6. A trend was seen toward a worse disease free survival for patients with TSR-high tumors compared to patients with TSR-low tumors. evaluating the stroma, the area of necrosis, the previous biopsy area, mucinous areas, and peripheral sides of tumors should not be evaluated. Therefore, the pathologist has a role in the selection of the appropriate tissue in digital analyzes. Digital methods are also not readily available, and extra costs are required to use them. For this reason, our study is based on visual eyeballing evaluation with light microscopy, which is very practical and does not require extra cost. Also, in our study, high intra-observer agreement kappa values for TSR prove strongly that TSR is a highly reproducible method.

Recent studies have mainly worked on TSR of triple-negative breast cancers, which are negative for ER, PR, and HER2. Vangangelt et al. reported that a high amount of stroma predicts poor survival in TN BC. Kruijf et al. showed TSR to be an independent prognostic factor for DFS in breast cancer patients, especially in those with TN BC. Also, Dekker et al. confirmed this finding by a validation study in the EORTC peri-operative chemotherapy trial ${ }^{18}$. The relationship between molecular subtypes and TSR was evaluated in our study, but no statistically significant relationship was found ( $\mathrm{p}>0.05$ ). This result supports the idea that the molecular properties of tumor cells are independent of the molecular properties of the tumor stroma.

In this study, TSR was found to be associated with lymph node metastasis, which is a prognostic factor independent of clinicopathological parameters. The incidence of lymph node metastasis increases in patients with TSR high $(\mathrm{p}=0.004)$ compared to patients with TSR low. When the overall survival was evaluated according to TSR high and TSR low among 363 patients whose survival information was available, the OS of the group with TSR high was significantly shorter than TSR low $(\mathrm{p}=0.002)$ patients. Also, in univariate $(\mathrm{p}=0.003)$ and multivariate cox regression models, TSR was an independent prognostic variable for OS.

Tumor-stroma ratio was also examined in node-positive Luminal A and B groups to evaluate the relationship between TSR and survival in a more homogeneous group. The overall survival of this group with TSR high was significantly shorter than TSR low $(p<0.001)$. Also, in univariate $(p=0.003)$ and multivariate cox regression models, the TSR was an independent prognostic variable for OS. TSR is an independent risk factor in this group of patients whose survival may differ. It is a parameter that can be used to determine prognosis. A potential limitation of our study was that patients with a short follow-up period were also included in this study in order to evaluate more patients. This may be the reason why TSR and DFS are not associated.

Adjuvant chemotherapy is controversial in early-stage TN and ER-positive BC. 17th St. Gallen International Breast Cancer Conference suggested genomic assays in addition to 
clinicopathological parameters in patient selection for treatment in PT1/PT2 N0 ER-positive patients ${ }^{10}$. However, in the same panel, it was reported that these genomic assays are not easily accessible universally and cost much more than routine pathology procedures. In our study, we showed that TSR is a decisive, independent prognostic factor. Therefore, TSR may be a parameter in the treatment decision, especially in this group of patients who have difficulties making treatment decisions.

There are publications in the literature reporting that clinical features are also effective in prognosis in BCs, regardless of the histological type $\mathrm{e}^{20-22}$. One of the limitations of this study is that clinical features were not evaluated. Another limitation is that the molecular and histological types of the cases are not homogeneously distributed.

\section{CONCLUSION}

Tumor-stroma ratio is an independent prognostic parameter that can be evaluated quite easily in all molecular subtypes of

\section{REFERENCES}

1. Plava J, Cihova M, Burikova M, Matuskova M, Kucerova L, Miklikova S. Recent advances in understanding tumor stroma-mediated chemoresistance in breast cancer. Mol Cancer. 2019;18(1):67. https://doi.org/10.1186/s12943-019-0960-z

2. Bandyopadhyay S, Bluth MH,Ali-Fehmi R. Breastcarcinoma: updates in molecular profiling 2018. Clin Lab Med. 2018;38(2):401-20. https://doi.org/10.1016/j.cll.2018.02.006

3. Majidinia M, Yousefi B. Breast tumor stroma: a driving force in the development of resistance to therapies. Chem Biol Drug Des. 2017;89(3):309-18. https://doi.org/10.1111/cbdd.12893

4. Ahn S, Cho J, Sung J, Lee JE, Nam SJ, Kim KM, et al. The prognostic significance of tumor-associated stroma in invasive breast carcinoma. Tumour Biol. 2012;33(5):1573-80. https://doi.org/10.1007/ s13277-012-0411-6

5. Hanahan D. Coussens LM. Accessories to the crime: functions of cells recruited to the tumor microenvironment. Cancer Cell. 2012;21(3):309-22. https://doi.org/10.1016/j.ccr.2012.02.022

6. Zhang $\mathrm{T}, \mathrm{Xu}$ J, Shen $\mathrm{H}$, Dong W, Ni Y, Du J. Tumor-stroma ratio is an independent predictor for survival in NSCLC. Int J Clin Exp Pathol. 2015;8(9):11348-55. PMID: 26617859

7. Zong L, Zhang Q, Kong Y, Yang F, Zhou Y, Yu S, etal. The tumor-stroma ratio is an independent predictor of survival in patients with 2018 FIGO stage IIIC squamous cell carcinoma of the cervix following primary radical surgery. Gynecol Oncol. 2020;156(3):676-81. https://doi.org/10.1016/j.ygyno.2019.12.022

8. Kramer CJH, Vangangelt KMH, Pelt GW, Dekker TJA, Tollenaar RAEM, Mesker WE. The prognostic value of tumour-stroma ratio in primary breast cancer with special attention to triple-negative tumours: a review. Breast Cancer Res Treat. 2019;173(1):55-64. https://doi.org/10.1007/s10549-018-4987-4 all BCs and does not require extra cost and time to evaluate. Therefore, TSR is a candidate practical parameter that can be included in routine pathological reports.

\section{ETHICS}

This study was conducted at Recep Tayyip Erdogan University Research and Training Hospital, Rize, Turkey, and conducted in accordance with the Declaration of Helsinki. The Ethics Committee approved the study protocol of Recep Tayyip Erdogan University.

\section{AUTHORS' CONTRIBUTIONS}

ÇÖ, OO: Conceptualization, Investigation, Visualization, Project administration. ÇÖ, OO, BŞ: Data curation, Writing - orginal draft. ÇÖ, BŞ: Formal Analysis, Methodology. RB: Funding acquisition, Validation. ÇÖ, RB: Resources. BŞ: Software. OO, RB: Supervision. OO, BŞ, RB: Writing - review \& editing.

9. Downey CL, Simpkins SA, White J, Holliday DL, Jones JL, Jordan LB, et al. The prognostic significance of tumour-stroma ratio in oestrogen receptor-positive breast cancer. Br J Cancer. 2014;110(7):1744-7. https://doi.org/10.1038/bjc.2014.69

10. Burstein HJ, Curigliano G, Loibl S, Dubsky P, Gnant M, Poortmans $\mathrm{P}$, et al. Estimating the benefits of therapy for early-stage breast cancer: the St. Gallen International Consensus Guidelines for the primary therapy of early breast cancer 2019. Ann Oncol. 2019;30(10):1541-57. https://doi. org/10.1093/annonc/mdz235

11. Mesker WE, Junggeburt JM, Szuhai K, Heer P, Morreau H, Tanke $\mathrm{HJ}$, et al. The carcinoma-stromal ratio of colon carcinoma is an independent factor for survival compared to lymph node status and tumor stage. Cell Oncol. 2007;29(5):387-98. https://doi. org/10.1155/2007/175276

12. Rakha EA, Allison KH, Ellis IO, Horii R, Masuda S, Penault-Llorca F, et al. Invasive breast carcinoma: general overview. In: Lokuhetty D, White VA, Watanabe R, Cree IA, editors. Breast tumours, WHO classification of tumours. 5th ed. Lyon: IARC; 2019. p. 82-101.

13. Bussard KM, Mutkus L, Stumpf K, Gomez-Manzano C, Marini FC. Tumor-associated stromal cells as key contributors to the tumor microenvironment. Breast Cancer Res. 2016;18(1):84. https:// doi.org/10.1186/s13058-016-0740-2

14. Dvorak HF. Tumors: wounds that do not heal. Similarities between tumor stroma generation and wound healing. N Engl J Med. 1986;315(26):1650-9. https://doi.org/10.1056/ NEJM198612253152606

15. College of American Pathologists (CAP). Protocol for the examination of biopsy specimens from patients with invasive carcinoma of the breast. 2020.[Online]. Available from: https:// www.cap.org/protocols-and-guidelines/cancer-reporting-tools/ cancer-protocol-templates. 
16. Vangangelt KMH, Green AR, Heemskerk IMF, Cohen D, Pelt GW, Sobral-Leite M, etal. The prognostic value of the tumor-stroma ratio is most discriminative in patients with grade III or triple-negative breast cancer. Int J Cancer. 2020;146(8):2296-304. https://doi. org/10.1002/ijc.32857

17. Kruijf EM, Nes JG, Velde CJ, Putter H, Smit VT, Liefers GJ, et al. Tumor-stroma ratio in the primary tumor is a prognostic factor in early breast cancer patients, especially in triplenegative carcinoma patients. Breast Cancer Res Treat. 2011;125(3):687-96. https://doi.org/10.1007/s10549010-0855-6

18. Dekker TJ, Velde CJ, Pelt GW, Kroep JR, Julien JP, Smit $V T$, et al. Prognostic significance of the tumor-stroma ratio: validation study in node-negative premenopausal breast cancer patients from the EORTC perioperative chemotherapy (POP) trial (10854). Breast Cancer Res Treat. 2013;139(2):371-9. https://doi.org/10.1007/s10549-0132571-5
19. Curigliano G, Burstein HJ, Winer EP, Gnant M, Dubsky P, Loibl S et al. De-escalating and escalating treatments for early-stage breast cancer: the St. Gallen International Expert Consensus Conference on the Primary Therapy of Early Breast Cancer 2017. Ann Oncol. 2017;28(8):1700-12. https://doi.org/10.1093/annonc/mdx308

20. Gomes da Cunha JP, Goncalves R, Silva F, Aguiar FN, Mota BS, Chequim BB, et al. Validation of the Residual Cancer Burden Index as a prognostic tool in women with locally advanced breast cancer treated with neoadjuvant chemotherapy. J Clin Pathol. 2021. https://doi.org/10.1136/jclinpath-2021-207771

21. Souza MA, Fonseca AM, Bagnoli VR, Barros N, Neves EM, Moraes $\mathrm{SD}$, et al. The expression of the estrogen receptor in obese patients with high breast density (HBD). Gynecol Endocrinol. 2014;30(1):7880. https://doi.org/10.3109/09513590.2013.859669

22. Trinconi AF, Filassi JR, Soares JM Jr, Baracat EC. Evaluation of the insulin-like growth factors (IGF) IGF-I and IGF binding protein 3 in patients athigh risk for breastcancer. Fertil Steril.2011;95(8):2753-5. https://doi.org/10.1016/j.fertnstert.2011.02.014 